\title{
Biomechanically Based Elastic Breast Registration Using Mass Tensor Simulation
}

\author{
Liesbet Roose, Wouter Mollemans, Dirk Loeckx, Frederik Maes, \\ and Paul Suetens \\ Medical Image Computing (Radiology - ESAT/PSI), \\ Faculties of Medicine and Engineering, University Hospital, Gasthuisberg, \\ Herestraat 49, B-3000 Leuven, Belgium \\ liesbet.roose@uz.kuleuven.ac.be
}

\begin{abstract}
We present a new approach for the registration of breast MR images, which are acquired at different time points for observation of lesion evolution. In this registration problem, it is of utmost importance to correct only for differences in patient positioning and to preserve other diagnostically important differences between both images, resulting from anatomical and pathological changes between both acquisitions. Classical free form deformation algorithms are therefore less suited, since they allow too large local volume changes and their deformation is not biomechanically based. Instead of adding constraints or penalties to these methods in order to restrict unwanted deformations, we developed a truly biomechanically based registration method where the position of skin and muscle surface are used as the only boundary conditions. Results of our registration method show an important improvement in correspondence between the reference and the deformed floating image, without introducing physically implausible deformations and within a short computational time.
\end{abstract}

\section{Introduction}

Breast cancer is the most common cancer in women worldwide, accounting for 1 million new cases annually [1. Currently, the detection and diagnosis of breast cancer primarily relies on projective X-ray mammography. In addition, contrastenhanced MRI images are acquired of women with dense breast tissue, for breasts with implants or scar tissue from prior surgery or in case of lobular cancer. Some patients undergo successive MRI scans with some months in between for followup of suspicious lesions.

Analysis and comparison of such follow-up images by the radiologist during diagnosis is greatly facilitated by proper geometric registration of the images. Image registration for breast imaging is a field of particular clinical interest, and in the last decade, many valuable approaches have been published. Zuo et al. 2. were the first to present a registration algorithm for the alignment of a pair of images acquired before and after contrast administration, but their approach is restricted to rigid transformations, whereas the soft tissue structure of the breast makes a non-rigid deformation model more appropriate. 
Several non-rigid registration techniques have been proposed and implemented for the registration of pre- and post-contrast breast MRI images, including algorithms based on the optical-flow algorithm [3], on elastic alignment of detected edges in the image 4 and in particular on a B-spline deformation as introduced by Rueckert et al. [5] using mutual information [6] as voxel-based similarity measure that is robust against local contrast changes in the pre- and post-contrast images. However, when assessing lesion evolution over time from a series of coregistered follow-up images, the degrees of freedom of the non-rigid registration method have to be constrained such that the method is able to compensate for global differences in patient positioning, but at the same time preserves local image changes induced by the pathology itself (e.g. tumor growth). While some authors imposed ad-hoc regularization penalties on the deformation field, for instance aimed at restricting local volume changes [7/8], we instead propose a truly biomechanical model where (near) volume preservation and elastic behavior is automatically fulfilled. Biomechanical breast models, mostly based on Finite Element Models (FEM), have been developed and used in different applications, for instance to predict the deformation of the breast during biopsy [9], for the registration of projective X-ray mammography and volumetric MRI images [10] or for the prediction of the effect of breast augmentation [11.

In this paper, we present a method for elastic intra-subject MR breast image registration based on a biomechanical deformation model implemented as a Mass Tensor Model. We illustrate our approach with results from 3 sets of patient data, showing an important improvement in correspondence between the reference and the deformed floating image, without introducing physically implausible deformation and in short computational time.

We use an implementation of the FEM-based Mass Tensor Model, introduced by Cotin et al. 12, which results in fast solution computations. Since we only want to recover the tissue changes due to different positioning of the breast in the imaging coil and different tension in the pectoralis major muscle, we choose the position of the skin and the muscle surface in the reference image as boundary constraint for the floating image. The deformation is thus only driven by the position of the segmented skin and muscle surface and not by the grey values in the rest of the image. A new equilibrium is then computed for the breast model subject to this boundary constraints and the floating image is deformed according to the deformation of the breast model.

\section{Method}

\subsection{Image Preprocessing}

To initialize the position of the floating image $F$ relative to the reference image $R$, a rigid registration algorithm is applied first to the two images [6]. Based on the transformed floating image, a volumetric tetrahedral mesh representation of the breast tissue is constructed, which is needed to perform the FEM computations. To this end, the breast tissue (skin, fat and fibroglandular tissue) is first segmented out of the MR image by thresholding, followed by a smoothing of 


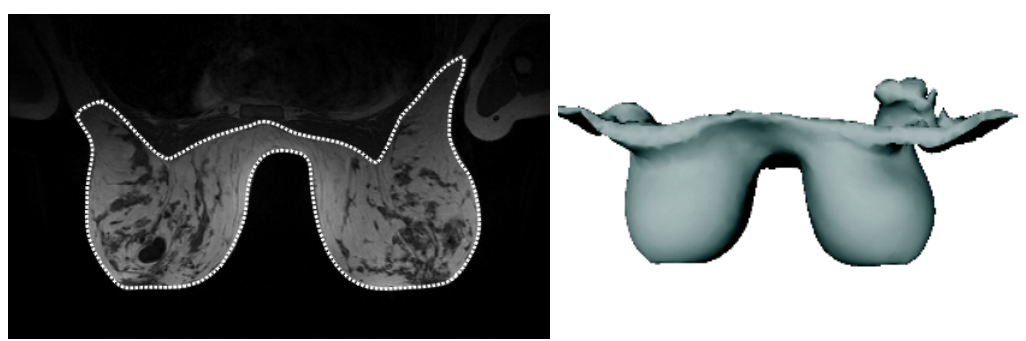

Fig. 1. Left: Slice of an original MRI image, with breast tissue segmented. Right: Three dimensional surface rendering of the resulting mesh of the breast tissue.

the segmented volume and removal of islands classified as background inside the breast region (Fig. 1). However, in some slices this simple segmentation scheme still needs manual correction afterwards.

The segmented volume is then converted to a tetrahedral volume mesh (Fig. 11). To assign boundary conditions to the floating image in the registration phase, skin and muscle surface need to be defined in the reference images. Therefore, after segmentation of the reference image, the skin and muscle surface in this image need to be indicated and are stored as triangular surface meshes.

\subsection{Biomechanial Transformation Model}

Floating image $F$ needs to be registered to reference image $R$ by applying a transformation $\phi: \mathbb{R}^{3} \rightarrow \mathbb{R}^{3}$, such that $F^{\prime}(\mathbf{x})=F(\phi(\mathbf{x}))$ corresponds to image $R$. For the transformation model $\phi$, we use a biomechanically based finite element model. We follow the general approach proposed by Cotin et al. [12] to define internal forces at each vertex of the mesh, based on linear elasticity theory. This force $\mathbf{F}_{i}$ for vertex $i$ at position $\mathbf{P}_{i}$ (having as original position $\mathbf{P}_{i}^{0}$ ) with the set of neighbouring points $N(i)$ can be computed as

$$
\mathbf{F}_{i}=\left[K_{i i}\right]\left(\mathbf{P}_{i}^{0}-\mathbf{P}_{i}\right)+\sum_{j \in N(i)}\left[K_{i j}\right]\left(\mathbf{P}_{j}^{0}-\mathbf{P}_{j}\right)
$$

where the $\left[K_{i j}\right]$ are tensors which are completely determined by the rest position and the material characteristics of the tetrahedra adjacent to vertex $i$. They can therefore be computed once and reused during the simulation. According to the conclusions of Tanner 13, we use a homogeneous linear elastic material model for the breast with a Poisson coefficient of 0.45 (corresponding to almost incompressibility).

The general Newtonian equation of movement (with $m$ the mass)

$$
m \frac{d^{2} \mathbf{P}}{d t^{2}}=\gamma \frac{d \mathbf{P}}{d t}+\mathbf{F}
$$

is solved in an iterative quasi-static way: the position of a point at time $t+1$ is the solution of the static problem with boundary conditions given at time $t$. This 
makes the estimation of the damping coefficient $\gamma$ unnecessary. The iterations are stopped at iteration $t$ where the maximal displacement $\left\|\mathbf{P}_{i}^{t-1}-\mathbf{P}_{i}^{t}\right\|$ over all points drops below a threshold $D_{\text {stop }}$ :

$$
\max _{i}\left(\left\|\mathbf{P}_{i}^{t-1}-\mathbf{P}_{i}^{t}\right\|\right)<D_{\text {stop }}
$$

\subsection{Boundary Conditions}

The deformation of the finite element model is driven by boundary conditions applied to the vertices on the skin surface and on the muscle-fat interface, in order to align both surfaces in the floating and the reference images. Before the first iteration these boundary conditions are defined. First the skin surface is segmented in the reference image, then the skin surface in the floating image is registered rigidly with this surface using Iterative Closest Points 14 and finally all vertices on the skin surface in the floating image are projected to the nearest point on the skin surface of the reference image. The same procedure is repeated for the muscle surface. These displaced vertices remain fixed during the iterations, while the other vertices in the mesh converge to their equilibrium position.

\subsection{Deformation of the Floating Image}

The transformation function $\phi(\mathbf{x})$ can be written as $\mathbf{x}+\mathbf{u}(\mathbf{x})$ with $\mathbf{u}(\mathbf{x})$ denoting the displacement vector, parameterized by the displacements $\mathbf{P}_{i}^{0}-\mathbf{P}_{i}$ at each node of the mesh, as computed with the Mass Tensor simulation. If we write $T_{i}$ for the tetrahedron defined by the four vertices $T_{i}(j), j=0, \ldots, 3$, then the vector displacement inside this tetrahedron $T_{i}$ is defined as [12]:

$$
\mathbf{u}_{T_{i}}(\mathbf{x})=\sum_{j=0}^{3} b_{j}(\mathbf{x})\left(\mathbf{P}_{T_{i}(j)}^{0}-\mathbf{P}_{T_{i}(j)}\right)
$$

where $b_{j}(\mathbf{x})$ are barycentric coordinates of the point $\mathbf{x}$ inside the tetrahedron $T_{i}$. This transformation is applied to the floating image in order to obtain the final registered image.

\section{Results}

\subsection{Image Acquisition}

Our test dataset contains three pairs of MRI images, acquired with a 3-D FLASH sequence with $\mathrm{TR}=9 \mathrm{~ms}, \mathrm{TE}=4.7 \mathrm{~ms}$, flip angle $=25^{\circ}, \mathrm{FOV}=384 \mathrm{~mm}$ and axial slice orientation. The images have dimensions 384 x 384 x 64 with a voxel size of $1.04 \mathrm{~mm}$ x $1.04 \mathrm{~mm}$ x $2 \mathrm{~mm}$. Each pair of images contains two dynamic MRseries of the same patient (1 pre-contrast and 7 post-contrast acquisitions), taken at different times (with intervals of 6,5 en 17 months between both acquisitions for set 1, 2 and 3 respectively). For each pre-contrast pair, we registered the original image with the follow-up image. The segmented breast tissue is meshed with an average edge length of approximately $10 \mathrm{~mm}( \pm 200$ vertices, \pm 7500 tetrahedra). 


\subsection{Convergence Behavior}

To determine the convergence behavior of our iterative solution strategy, we repeatedly registered the images in set 1 for different values of $D_{\text {stop }}$ and compared the results with a solution computed up to machine accuracy. The results are shown in Fig. 2. From the analysis of the mean distance between corresponding nodes in the meshes, it can be seen that the error is monotonically decreasing, approximately linear with $\log \left(D_{\text {stop }}\right)$. The effect of this deviation of the node points on the transformed images is shown in the right graph of Fig. 2, showing the decrease in the total sum of squared differences (SSD) over the image and the number of voxels with different grey value.

All simulations have been carried out on a Pentium M, $1.7 \mathrm{GHz}, 1 \mathrm{~Gb}$ RAM, and the computation time is shown in the left graph of Fig. 2, showing computation times between $20 \mathrm{~s}$ for $D_{\text {stop }}=1 \mathrm{~mm}$ to $25 \mathrm{~min}$ for $D_{\text {stop }}=10^{-4} \mathrm{~mm}$. To reach a solution where maximum $1 \%$ of the voxels is different from the final solution, $D_{\text {stop }}$ is chosen at $0.005 \mathrm{~mm}$ corresponding with a computation time of approximately 90 seconds.
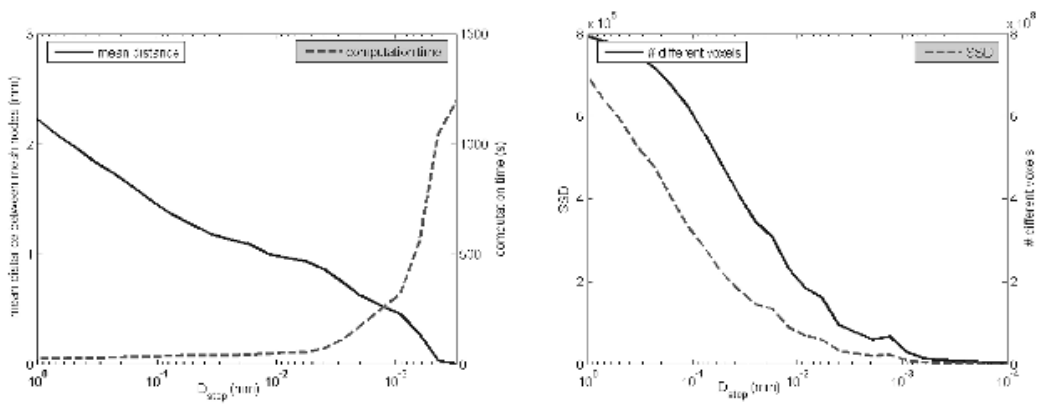

Fig. 2. Left: Computation time and mean error between the solution computed up to machine accuracy and solutions with different values of $D_{\text {stop }}$. Right: Difference in terms of sum of squared differences (SSD) and number of voxels with different grey values between this solution computed up to machine accuracy and solutions with different values of $D_{\text {stop }}$.

\subsection{Experimental Data}

To indicate the feasibility and usefulness of our method, we applied it to the three sets of patient data and compared the result of our non-rigid registration with the non-registered image, with a purely rigid registered image and with an intensity-based image registration using a spline-based deformation model [15. Fig. 3 shows the registered images, the difference images between (registered) floating and the reference image and the deformations for one case. It can be seen that our non-rigid registration gives a better alignement than the purely rigid registration alone, for example in locating the black cyst underneath the nipple in the breast on the left of the image. The deformed raster in the last column shows that the deformation is smooth and without important volumetric changes. The 
(1)
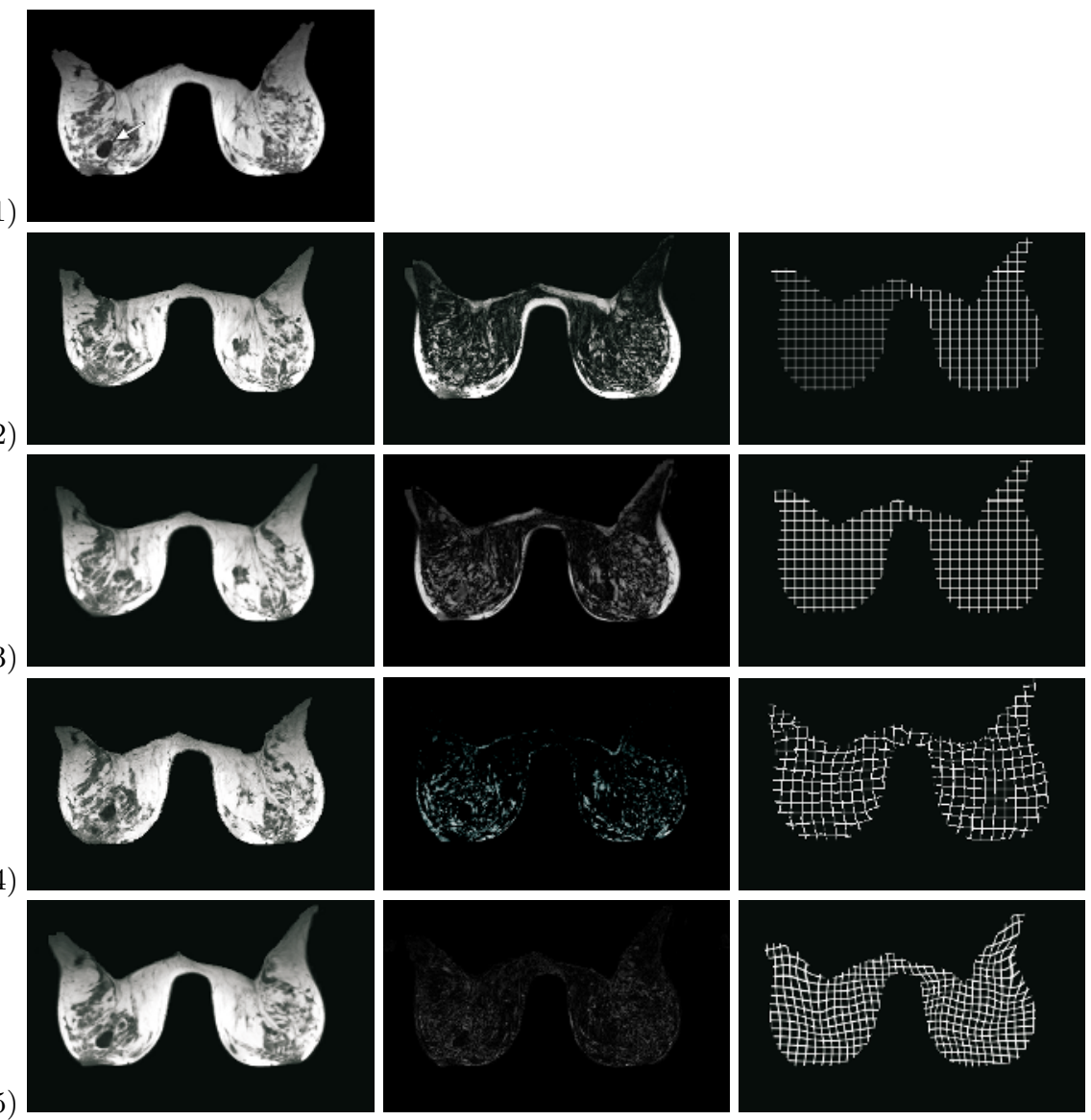

Fig. 3. Comparison of different registration techniques on a pair of breast images of the same patient, acquired with 6 months time in between. Registration is done on the segmented images, where only the breast tissue is preserved. The first row shows the reference image (with the lesion of interest indicated with the arrow), row 2, 3, 4 and 5 show respectively the floating image before registration, after rigid registration, after our non-rigid registration and after classical spline-based registration. The first column of row 2-5 shows the deformed floating image, the second column shows the difference image between floating and reference image (limited to the breast region) and the third column shows a regular raster pattern after application of the same deformation as the floating image, to illustrate the extent of deformation. Local areas of volume compression and stretching can be seen in the deformation field of the spline-based registration, whereas the deformation of our non-rigid registration is almost volume preserving.

classical spline-based algorithm gives a better alignment than our approach, but from the image of the deformation field, it can be seen that the deformation is physically not plausible for the nearly incompressible breast tissue and that the image is therefore not useful for diagnosis. Fig. 4 shows the reduction in SSD after rigid and non-rigid registration. 


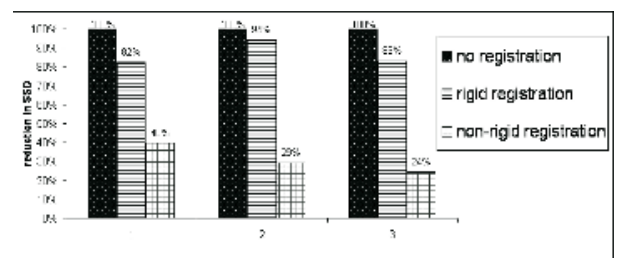

Fig. 4. The SSD (relative to the SSD of the non-registered images) for the three patients cases, without registration, with rigid registration and with our non-rigid registration

\section{Discussion and Conclusion}

This paper has introduced a biomechanically based registration method for the registration of MRI images of the breast, taken at different time points. Contrary to the registration of images taken in one image sequence, we expect the two images to be aligned to contain major anatomical differences. The use of classical gray-value based free form deformation algorithms is therefore not appropriate for this type of images, because of the risk of removing diagnostically important features from the image. Instead of adding extra constraints to free form methods, we implemented a FEM-based model where elastic properties and compressibility behavior of the breast tissue are automatically incorporated in the model. Since we only want to correct for different patient positioning in the imaging coil and different tension of the pectoralis major muscle, we only use the skin and muscle surface as boundary constraints. The algorithm has been demonstrated on three sets of patient data, showing its ability to reduce the SSD with $70 \%$ on average, without introducing biomechanically non realistic deformations.

Future works on this algorithm include the design of a more robust segmentation scheme and the coupling of segmentation, meshing and simulation to make our method fully automatic. Furthermore, we want to investigate the effect of changing the currently used boundary condition (connecting points to skin or muscle surface) to sliding boundary conditions (where the skin nodes of the floating image have a sliding contact with the skin surface of the reference image). Further experiments will be carried out to compare our algorithm with other non-rigid registration algorithms, with and without additional constraints.

\section{References}

1. Stewart, B., Kleihues, P.: World cancer report. Oxford University Press (2003)

2. Zuo, C.S., Jiang, A., Buff, B.L., Mahon, T.G., Wong, T.: Automatic motion correction for breast MR imaging. Radiology 198 (1996) 903-906

3. Kumar, R., Asmuth, J.C., Hanna, K., Bergen, J.: Application of 3D registration for detecing lesions in magnetic resonance scans. In: Medical Imaging: Image Processing, SPIE Press (1996) 
4. Lucht, R., Knopp, M.V., Brix, G.: Elastic matching of dynamic MR mammographic images. Magnetic Resonance in Medicine 43 (2000) 9-16

5. Rueckert, D., Sonoda, L., Hayes, C., Hill, D., Leach, M., Hawkes, D.: Nonrigid registration using free-form deformations: application to breast MR images. IEEE Trans. Medical Imaging (1999)

6. Maes, F., Collignon, A., Vandermeulen, D., Marchal, G., Suetens, P.: Multimodality image registration by maximization of mutual information. IEEE Trans. Medical Imaging 16 (1997) 187-198

7. Rohlfing, T., jr Maurer, C., Bluemke, D., Jacobs, M.: Volume-preserving nonrigid registration of MR breast images using free-form deformation with an incompressibility constraint. IEEE Trans Medical Imaging 22 (2003) 730-41

8. Loeckx, D., Maes, F., Vandermeulen, D., Suetens, P.: Nonrigid image registration using free-form deformations with a local rigidity constraint. In: Medical Image Computing and Computer-Assisted Intervention. (2004) 639-646

9. Azar, F.S., Metaxas, D.N., Schall, M.D.: Methods for modeling and predicting mechanical deformations of the breast under external pertubations. Med. Image Anal. 6 (2002) 127

10. Ruiter, N.: Registration of X-ray mammograms and MR-volumes of the female breast based on simulated mammographic deformation. PhD thesis, Universitaet Mannheim (2003)

11. Roose, L., Maerteleire, W.D., Mollemans, W., Suetens, P.: Validation of different soft tissue simulation methods for breast augmentation. In Lemke, H.U., ed.: Computer assisted radiology and surgery. Volume 1281 of ICS. (2005)

12. Cotin, S., Delingette, H., Ayache, N.: A hybrid elastic model allowing real-time cutting, deformations and force-feedback for surgery training and simulation. The Visual Computer 16 (2000) 437-452

13. Tanner, C., Degenhard, A., Schnabel, J., Smith, A., Hayes, C., Sonoda, L., Leach, M., Hose, D., Hill, D., Hawkes, D.: A method for the comparison of biomechanical breast models. Proceedings of the IEEE Workshop on Mathematical Methods in Biomedical Image Analysis (2001) 11-18

14. Besl, P.J., McKay, N.D.: A method for registration of 3-D shapes. IEEE Trans. Pat. Anal. and Mach. Intel. 14 (1992) 239-256

15. Loeckx, D.: Automated nonrigid intra-patient image registration using B-splines. PhD thesis, Katholieke Universiteit Leuven (2006) 